\title{
Rats with Extended Access to Cocaine Exhibit Increased Stress Reactivity and Sensitivity to the Anxiolytic-Like Effects of the mGluR 2/3 Agonist LY379268 during Abstinence
}

\author{
Harinder Aujla*', Rémi Martin-Fardon' and Friedbert Weiss' \\ 'Molecular and Integrative Neurosciences Department, The Scripps Research Institute, La Jolla, CA, USA
}

\begin{abstract}
Metabotropic glutamate $2 / 3$ receptors (mGluR2/3) are emerging targets for the reduction of stress that contributes to drug relapse. The effect of a history of cocaine escalation on stress reactivity during abstinence and the role of mGlu2/3 receptors in stress in these animals were tested. Experiment I - Rats trained to self-administer cocaine, under short (ShA, I-h) or long (LgA, 6-h) access conditions, or noncaloric food pellets (Ctrl, I-h), were tested for stress reactivity in the shock-probe defensive burying test following I, I4, 42, or 84 days of abstinence. Experiment 2 - Experimentally naive rats receiving the mGlu2/3 receptor agonist LY379268 (0, 0.3, 1.0, or 3.0 mg/kg) were tested in the defensive burying test to establish the anxiolytic efficacy of this compound in this model. Experiment 3-Rats with a history of ShA vs LgA cocaine self-administration, or a history of operant responding reinforced by noncaloric food pellets, were tested in the defensive burying test, following administration of LY379268 $(0.3,1.0$, or $3.0 \mathrm{mg} / \mathrm{kg})$ at 14 days of abstinence. $L g A$ rats exhibited a two- to threefold increase in defensive burying at I, 14, and 42 days of abstinence compared to ShA or control animals. LY379268 $(3.0 \mathrm{mg} / \mathrm{kg})$ reduced burying in all groups, whereas the $1.0-\mathrm{mg} / \mathrm{kg}$ dose reduced burying only in the LgA group. A robust and enduring increase in stress reactivity developed in rats with a history of daily 6-h access to cocaine. The anxiolytic-like effects of LY379268 identify mGlu2/3 receptors as targets for ameliorating stress-associated relapse risk, and point toward the possibility that a history of cocaine escalation in rats may modify glutamatergic function.

Neuropsychopharmacology (2008) 33, I8I8- |826; doi: I0.1038/s..npp. I 301588; published online 26 September 2007
\end{abstract}

Keywords: cocaine; escalation; defensive burying; stress; abstinence; metabotropic glutamate

\section{INTRODUCTION}

Termination of cocaine use in addicts is associated with heightened susceptibility to stress, which is ameliorated by resumption of drug use (Sinha, 2001). Heightened stress in abstinent cocaine users is associated with increased drug craving (Fox et al, 2005; Sinha, 2001), and exposure to acute stress during abstinence reliably elicits cocaine seeking in animal models of relapse (Erb et al, 1996, 2001; Sorge and Stewart, 2005). Moreover, stress contributes to the development of sensitization to cocaine (Post and Rose, 1976; Prasad et al, 1995; Meaney et al, 2002) and amphetamine (Antelman et al, 1980). Thus, stress is a significant risk factor in both the initiation of drug taking in drug-naive subjects, and relapse to drug use in abstinent cocaine addicts. Given that stress is a major risk factor for relapse,

*Correspondence: Dr H Aujla, Molecular and Integrative Neurosciences Department, The Scripps Research Institute, SP30-2120, 10550 North Torrey Pines Road, La Jolla, CA 92037, USA, Tel: + I 858784 7233, Fax: + I 858784 7I46, E-mail: haujla@scripps.edu Received 6 March 2007; revised 20 August 2007; accepted 20 August 2007 pharmacotherapies with anxiety-reducing actions may be beneficial in reducing relapse rates.

A promising target for the development of agents with anxiolytic-like effects is the group II family of metabotropic glutamate receptors (mGluRs), comprised of the mGluR2 and mGluR3 receptor subtypes. Previous studies have illustrated anxiolytic-like effects for the selective mGlu2/3 receptor agonist LY354740 - an agent that functionally reduces glutamatergic transmission - in the elevated plus maze test (Linden et al, 2005a). Also, the mGlu2/3 receptor agonist LY379268 has been shown to attenuate platform stress-evoked norepinephrine release in the medial prefrontal cortex (PFC) (Lorrain et al, 2005). The significance of group II mGluRs in mediating these 'antistress' effects is supported by the finding that the anxiolytic-like effects of LY354740 in the elevated plus maze are not observed in mGluR2 or mGluR3 knockout mice (Linden et al, 2005a).

Drug addiction is a chronically relapsing disorder. Recent animal studies have indicated pharmacotherapeutic potential of the mGlu2/3 receptor agonist LY379268 in treating relapse risk associated with cocaine (Peters and Kalivas, 2006; Adewale et al, 2006), drug-associated stimuli (Baptista et al, 2004; Bossert et al, 2004, 2006; Zhao et al, 2006), and 
stressful stimuli (Zhao et al, 2006). However, an important question is to what extent chronic drug use induces increased susceptibility to stress and thereby contributes to the maintenance or resumption of drug use. There is evidence that a history of chronic cocaine exposure increases stress reactivity during abstinence in both humans (Breese et al, 2005; Fox et al, 2005) and rats (Mutschler and Miczek, 1998a; Basso et al, 1999; Harris et al, 2001) that may contribute to relapse associated with stress. Previous studies have shown that subchronic noncontingent administration of cocaine produces stress-like effects as measured in the defensive burying test (Basso et al, 1999; Harris et al, 2001). However, it is not known whether chronic cocaine selfadministration produces a similar elevation in stress reactivity and, more importantly, whether a history of escalated cocaine self-administration (ie a model of cocaine addiction; Ahmed and Koob, 1998) further exacerbates stress reactivity.

Self-administration of cocaine (Bozarth and Wise, 1985; Johanson et al, 1976) or amphetamine (Yokel and Pickens, 1973), when conducted under unlimited access conditions, leads to an upregulation of drug intake. Particularly, in the 'escalation' model of dependence, rats receiving long daily access ( $\operatorname{LgA}$ ) to cocaine (typically $6 \mathrm{~h}$ ) show a progressive increase in daily drug intake over time (escalation), and show an upward shift in the cocaine dose-effect function (Ahmed and Koob, 1998, 1999) that reflects a shift in the hedonic set point (ie the need for markedly increased amounts of the substance to achieve the desired effect). Following termination of extended daily access to drug, cocaine-escalated rats suffer long-lasting reward deficit$\mathrm{s}$ - reflected by increased brain stimulation reward thresholds (Ahmed et al, 2002) - that may be analogous to depression and dysphoria that accompany withdrawal in cocaine addicts (eg Markou and Koob, 1991). Moreover, these animals, in the case of heroin, exhibit heightened susceptibility to stress-induced relapse (Ahmed et al, 2000). Thus, the escalation model provides an opportunity to study the behavioral and neurobiological effects of cocaine under conditions that mimic the consequences of chronic cocaine abuse in humans.

The purpose of the present investigation was to (1) examine the long-term effects on stress reactivity in rats with and without a history of escalated cocaine selfadministration at increasing delays following termination of access to cocaine and (2) evaluate the anxiolytic-like effects of LY379268 in rats with different histories of cocaine self-administration. Stress reactivity was measured using the shock-probe defensive burying test (Pinel and Treit, 1978 ) in which the primary index of stress or anxiety is the duration spent by rats burying an electrified probe, with increased burying time providing a measure of elevated stress reactivity or anxiety. Clinically effective anxiolytic agents such as diazepam, chlordiazepoxide, and buspirone attenuate levels of defensive burying (De Boer and Koolhaas, 2003), suggesting that this test is a useful tool for evaluating the anxiolytic potential of novel agents. Burying tests were conducted $1,14,42$, or 84 days following termination of access to cocaine. Rats trained to selfadminister noncaloric food pellets or cocaine under nonescalation conditions served as controls. The anxiolytic profile of LY379268 on shock probe-induced defensive burying was measured at a single time point (14 days) following discontinuation of access to cocaine or food pellet reinforcers. Additionally, the effects of LY379268 on defensive burying were examined in rats without a history of either drug or food reinforcement to control for any potential effects of reinforcement history.

\section{MATERIALS AND METHODS}

\section{Subjects}

Male Wistar rats (Charles River, Wilmington, MA), weighing 200-250 g on arrival, were housed in groups of 2-3 on a 12-h (lights off at 0800) light/dark cycle in a temperatureand humidity-controlled vivarium. Food and water were available ad libitum except during the food training sessions conducted before catheter implantation. All behavioral training and testing sessions were initiated during the rats' dark cycle. All procedures were conducted in strict adherence to the National Institutes of Health Guide for the Care and Use of Laboratory Animals.

\section{Self-Administration Apparatus}

Cocaine self-administration was conducted in standard $29 \times 24 \times 19.5 \mathrm{~cm}$ operant conditioning chambers located inside ventilated sound-attenuating cubicles (BRS/LVE Inc., Laurel, MD), modified for intravenous self-administration as previously described (Weiss et al, 2000). All chambers were equipped with two retractable levers located $6 \mathrm{~cm}$ above the grid floor. A white cue light $(24 \mathrm{~W})$ was positioned $4 \mathrm{~cm}$ above each lever. Intravenous infusions were administered by an automated syringe pump (Harvard Apparatus, Holliston, MA) located outside the soundattenuating cubicles. Syringes $(10 \mathrm{ml})$ were connected via Tygon tubing and a cannula connector (C3136, Plastics One, Roanoke, VA) to a chronic jugular catheter on the rats' backs. Testing equipment and data collection were controlled by an IBM-compatible microcomputer.

\section{Defensive Burying Apparatus}

A standard polycarbonate cage $(43 \times 20 \times 20 \mathrm{~cm})$ containing a 5-cm layer of bedding material (wood shavings) was used. For testing, an electrical shock probe (Staco Energy Products, Dayton, $\mathrm{OH}$ ) was inserted through a small diameter opening $(2 \mathrm{~cm})$ located $2.5 \mathrm{~cm}$ above the bedding in the center of the chamber's long side. The probe protruded $6.5 \mathrm{~cm}$ into the cage and was connected to a Coulbourn precision shocker (model E13-01, Allentown, PA), calibrated to deliver $1.5 \mathrm{~mA}$ of shock when contacted.

\section{Drugs}

Cocaine hydrochloride (National Institute on Drug Abuse, Bethesda, MD) was dissolved in sterile physiological saline to a concentration of $2.5 \mathrm{mg} / \mathrm{ml}$. LY379268 (provided by Dr D Shoepp, Eli Lilly) was dissolved in sterile water to a concentration of $0.3,1.0$, or $3.0 \mathrm{mg} / \mathrm{ml}$ (weight of salt).

Experiment 1 -Defensive burying during abstinence from short or long access to cocaine. 


\section{Cocaine Self-Administration}

Rats $(n=73)$ were transiently placed under food restriction (Purina chow $15 \mathrm{~g} / \mathrm{d}$ ) and trained to lever press for $45-\mathrm{mg}$ noncaloric cellulose pellets (Research Diets, New Brunswick, NJ) under a fixed-ratio (FR) 1 schedule until a criterion of at least 60 pellets/session was reached for three consecutive 1-h sessions. A time-out (TO) period of $20 \mathrm{~s}$, signaled by the illumination of a cue light located above the active lever, then was introduced. During the time-out period the lever remained inactive in preparation for later cocaine self-administration training where inactivation of the lever served to prevent accidental overdosing. Training continued under these contingencies until subjects reached a criterion of at least 60 pellets/session for two consecutive 1-h sessions.

Upon completion of food training, animals were returned to ad libitum feeding. Rats assigned to the short access (ShA; $n=25)$ and long access (LgA; $n=23$ ) cocaine conditions were surgically prepared with indwelling silastic catheters in the right jugular vein under 1.5-2\% isoflurane anesthesia as described previously (Caine and Koob, 1993). Catheter patency was maintained throughout the experiment by flushing with $0.1 \mathrm{ml}$ of sterile saline solution containing 33.3 USP units/ml of heparin before and after each self-administration session.

Cocaine self-administration training began after 7-10 days of recovery in daily 1 -h sessions. These sessions were initiated by extension of both the active and inactive levers. Responses at the active lever were reinforced under an FR1 schedule (with a 20-s cue light-signaled time-out) by an intravenous injection of cocaine $(0.25 \mathrm{mg}$ per injection) delivered over a 4 -s period in a volume of $0.1 \mathrm{ml}$. Responses at the inactive lever were recorded but had no scheduled consequences. Training continued until criterion performance $( \pm 10 \%$ over three consecutive sessions) was reached. Upon completion, rats were divided into two groups receiving either limited or long access to cocaine during the subsequent 'escalation' phase.

\section{Escalation}

Animals in the ShA group $(n=25)$ continued to receive daily access to cocaine in 1-h sessions under an FR1 TO 20-s schedule. Rats in the $\operatorname{LgA}$ group $(n=23)$ received daily access to cocaine in 6-h sessions under otherwise identical reinforcement contingencies as the ShA group. Self-administration under these conditions was conducted daily until completion of 22 sessions.

\section{Cocaine-Naive Controls (Ctrl)}

After acquisition of food-reinforced responding and being returned to ad libitum feeding, rats $(n=25)$ continued to receive response-contingent access to $45 \mathrm{mg}$ noncaloric cellulose pellets under an FR1 TO 20-s schedule in daily $1 \mathrm{~h}$ sessions.

\section{Defensive Burying}

Each of the three treatment groups (ShA, LgA, and Ctrl) was subdivided into four groups for testing either $1,14,42$, or 84 days following termination of access to cocaine or food pellets. Rats were habituated to the testing environment and defensive burying apparatus (without insertion of the shock probe) on 2 consecutive days for a total of $5 \mathrm{~h}$ in the presence of white noise $(70 \mathrm{~dB}$; Realistic sound amplifier, model SA-10). On the third day, each animal was subjected to a 10-min test in the defensive burying apparatus under conditions identical to the habituation sessions, except for the introduction of the electrified probe. Upon contact with the probe and shock delivery $(<1 \mathrm{~s})$, verified by a startle response, the probe was deactivated and subsequent burying behavior was recorded by video camera for later scoring. Bedding was changed and the cage washed between tests. Video footage for each animal was later scored for the duration of (1) burying, (2) freezing, defined as rigid immobile posture, (3) ambulation, and (4) rearing. In addition, shock reactivity was scored on a four-point scale (Pesold and Treit, 1992), based on the startle response exhibited by the rat upon first contact with the electrified probe. The shock reactivity scale included the following measures: (1) flinch involving only the forepaw or head; (2) whole body flinch without ambulation away from the shock probe; (3) whole body flinch and/or jumping with ambulation away from the shock probe; and (4) whole body flinch and/or jumping followed by running to the opposite side of the test cage. This measure provides an index of the amount of pain produced by contact with the probe, a parameter that may influence burying behavior.

Experiment 2-The effects of LY379268 on defensive burying in naive animals.

Rats $(n=38)$ were treated subcutaneously with LY379268 doses of $0(n=9), 0.3 \mathrm{mg} / \mathrm{kg}(n=10), 1.0 \mathrm{mg} / \mathrm{kg}(n=10)$, or $3.0 \mathrm{mg} / \mathrm{kg}(n=9), 30 \mathrm{~min}$ before defensive burying sessions. All procedures for defensive burying habituation and test sessions were identical to those in Experiment 1.

Experiment 3-The effect of LY9268 on defensive burying in LgA, ShA, and Ctrl rats at 14 days of following the last self-administration session.

\section{Cocaine Self-Administration}

Rats $(n=75)$ received cocaine self-administration training and daily access to cocaine under the $\operatorname{LgA}(n=26)$, ShA $(n=26)$, and Ctrl $(n=23)$ conditions, following the same protocol as in Experiment 1. A 2-week drug-free period elapsed before the defensive burying test.

\section{Defensive Burying}

Rats in each drug-history group were treated with $0.3 \mathrm{mg} /$ kg. (LgA: $n=11$; ShA: $n=10$; Ctrl: $n=11), 1.0 \mathrm{mg} / \mathrm{kg}$ (LgA: $n=9$; ShA: $n=9$; Ctrl: $n=6)$, or $3.0 \mathrm{mg} / \mathrm{kg}$ (LgA: $n=6$; ShA: $n=7$ : Ctrl: $n=6$ ) of LY379268 subcutaneously $30 \mathrm{~min}$ before onset of defensive burying sessions. All habituation and test procedures were identical to those described in Experiment 1.

\section{Statistical Analysis}

Differences between the first and last self-administration session for each treatment group were analyzed via a paired $t$-test. Differences in cocaine-reinforced responding 
between the ShA and the LgA group were analyzed using a two-way mixed-factorial analysis of variance, with drug history as the between-subjects factor and self-administration session as the within-subjects factor, followed by Newman-Keuls post hoc tests to verify differences among individual means. Tests of normality using Wilkes-Shapiro analysis $(W=0.7943, p<0.05)$ as well as tests for homogeneity of variance using Bartlett's test $\left(K^{2}=6.6012\right.$, $p<0.05)$ revealed that the defensive burying data violated these assumptions. These data were therefore analyzed by nonparametric Kruskal-Wallis one-way tests for each time point (Experiment 1) or dose of LY379268 (Experiment 3) to evaluate differences among the Ctrl, ShA, and LgA groups. Similarly, a Kruskal-Wallis one-way test was used to evaluate the dose-effect function for LY379268 on defensive burying in Experiment 2. Significant omnibus tests were followed by Mann-Whitney $U$ pairwise comparisons to verify between-group differences. Shock reactivity scores were ordinal data and therefore analyzed using Kruskal-Wallis one-way tests followed by Mann-Whitney $U$ pairwise comparisons where appropriate for all experiments.

\section{RESULTS}

\section{Experiment 1}

Self-administration. Extending daily access to $6 \mathrm{~h}$ in the LgA group resulted in progressively increasing cocaine selfadministration with a significant difference between the last and first self-administration session in terms of both total $\left(\mathrm{t}_{22}=2.47 ; p<0.05\right)$ and first-hour $\left(\mathrm{t}_{22}=2.53 ; p<0.05\right)$ cocaine intake (Figure 1). In contrast, no difference between the first and last self-administration session was observed in the sha and ctrl groups (Figure 1). Moreover, scrutiny of the time course of responding during the escalation period revealed a significant increase only in the LgA group that was significant for the total session length, beginning at session $6(p<0.05)$ and for the total session as well as first-hour intake beginning at session 9 compared to

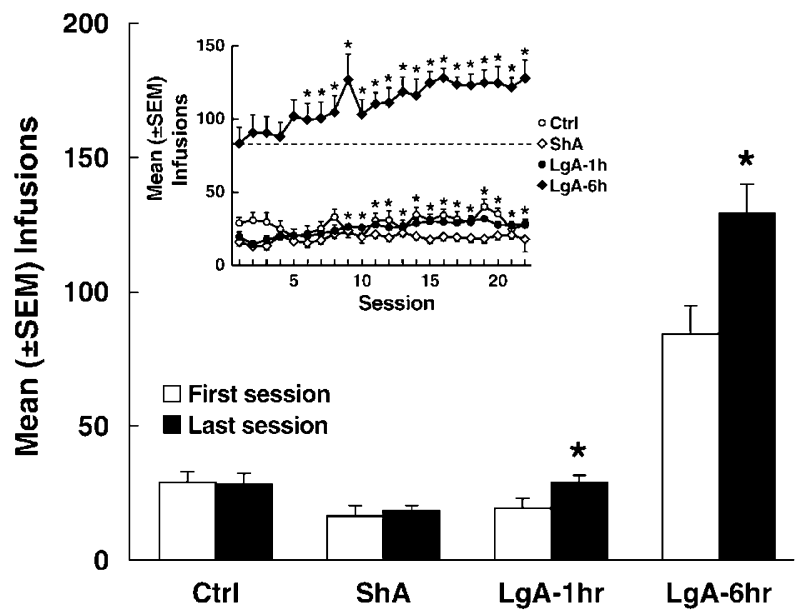

Figure I Number of lever presses on the first vs last self-administration session for food control (Ctrl), short access cocaine (ShA), and long access cocaine ( $\mathrm{LgA}-\mathrm{I} h$ and $\mathrm{LgA}-6 \mathrm{~h}$ ) rats ( $\mathrm{*} p<0.05$ with a paired $t$-test on first vs last self-administration session). Inset: number of lever presses on each day of self-administration corresponding to the escalation phase of the experiment for Ctrl, ShA, and LgA (I and $6 \mathrm{~h}$ ) rats (* $p<0.05$ vs session I). the first session (Figure 1 inset, Newman-Keuls post hoc test after significant session $\times$ access group interaction: $\left.\mathrm{F}_{3,63}=9.34 ; p<0.05\right)$.

Defensive burying. Overall, burying time in the shock-probe test was significantly increased in rats of the $\operatorname{LgA}$ group compared to both the ShA and Ctrl groups (Figure 2). This increase in burying time was confirmed by Kruskal-Wallis tests with significant group effects at the $1\left(\mathrm{H}_{2,18}=5.93\right.$, $p<0.05), 14\left(\mathrm{H}_{2,18}=8.06, p<0.05\right)$, and $42\left(\mathrm{H}_{2,17}=7.16\right.$, $p<0.05)$ day time points, but not at the 84 day time point (Figure 2). Mann-Whitney $U$ pairwise comparisons revealed that at day 1 of abstinence, $\operatorname{LgA}$ rats exhibited increased burying compared to the $\operatorname{ShA}(U=6, p<0.05)$ and Ctrl ( $U=5, p<0.05)$ groups, while the ShA and Ctrl groups did not differ from one another. Similarly, at 14 days of abstinence, LgA rats showed increased burying compared to ShA rats $(U=5, p<0.05)$ and $\operatorname{Ctrl}(U=3, p<0.05)$ without differences between the latter two groups. The same pattern of effects was observed at 42 days of abstinence, with significant differences between LgA and ShA rats $(U=2, p<0.05)$ as well as between $\operatorname{LgA}$ and Ctrl rats $(U=3, p<0.05)$, again with no differences between the ShA and Ctrl groups.

Other behavioral measures. No significant effects were observed for freezing, shock reactivity, ambulation, or rearing in the LgA group vs the ShA and Ctrl groups at any time point. In addition, no differences in these behaviors were observed between ShA and Ctrl groups at any time point (data not shown).

\section{Experiment 2}

Burying duration. Significant Kruskal-Wallis test $\left(\mathrm{H}_{3,38}=12.14, p<0.05\right)$ revealed an effect of LY379268 on burying duration (Figure 3 ). Mann-Whitney $U$ pairwise comparisons confirmed that a reduction in burying was observed at the $3.0 \mathrm{mg} / \mathrm{kg}(U=3, p<0.05)$ but not at the 0.3 or $1.0 \mathrm{mg} / \mathrm{kg}$ dose of LY379268 vs vehicle.

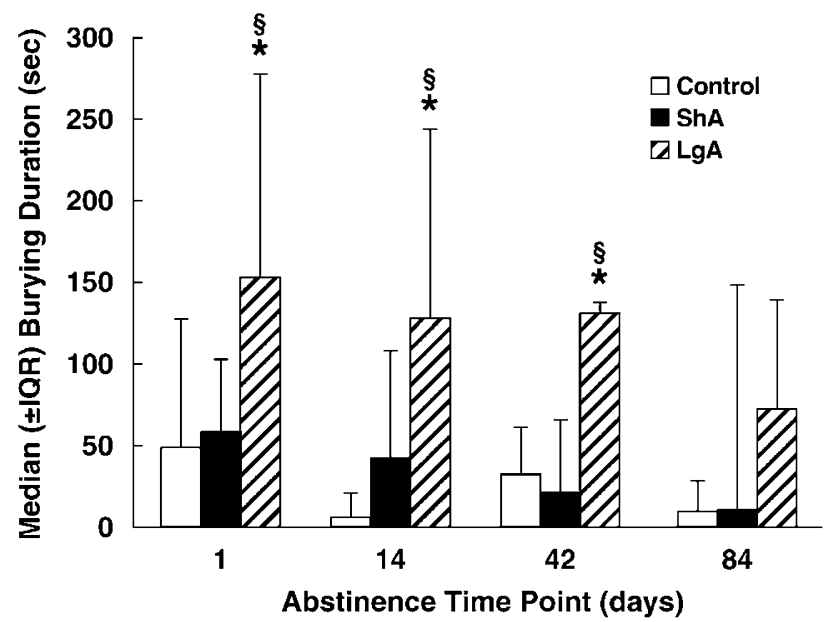

Figure 2 Time, in seconds, spent burying a previously electrified probe in the defensive burying test for food control (Ctrl), short access cocaine (ShA), and long access cocaine (LgA) rats at I, 14, 42, or 84 days from the last self-administration session (* $p<0.05$ with a Mann-Whitney U-test vs Ctrl, ${ }^{\S} p<0.05$ with a Mann-Whitney U-test vs ShA). 
Other behavioral measures. No significant effects were observed for freezing, shock reactivity, ambulation, or rearing in the LgA group $v s$ the ShA and Ctrl groups at any time point. In addition, no differences in these behaviors were observed between ShA and Ctrl groups at any time point (Table 1).

\section{Experiment 3}

Self-administration. Extending daily access to $6 \mathrm{~h}$ in the LgA group produced increasing cocaine self-administration with significant differences between the first and last selfadministration session in both total $\left(\mathrm{t}_{25}=2.34 ; p<0.05\right)$ and first-hour $\left(\mathrm{t}_{25}=2.26 ; p<0.05\right)$ cocaine intake (Figure 4$)$. No difference between the first and last self-administration sessions was observed in the ShA and Ctrl groups (Figure 4). Increased responding during the escalation period was observed only in the LgA group and was significant for firsthour intake beginning at session $11(p<0.05)$ and at session 12 for total session as well as first-hour intake $(p<0.05)$ compared to intake for the first session (Figure 4 inset, Newman-Keuls post hoc test after significant session $\times$ access condition interaction: $\left.\mathrm{F}_{3,63}=10.86 ; p<0.05\right)$.

Burying duration. A significant drug-history group effect for burying time was confirmed by Kruskal-Wallis tests at the $0.3 \mathrm{mg} / \mathrm{kg}\left(\mathrm{H}_{2,32}=7.84, p<0.05\right)$ but not at the $1.0 \mathrm{mg} / \mathrm{kg}$ or $3.0 \mathrm{mg} / \mathrm{kg}$ doses of LY379268 (Figure 5).

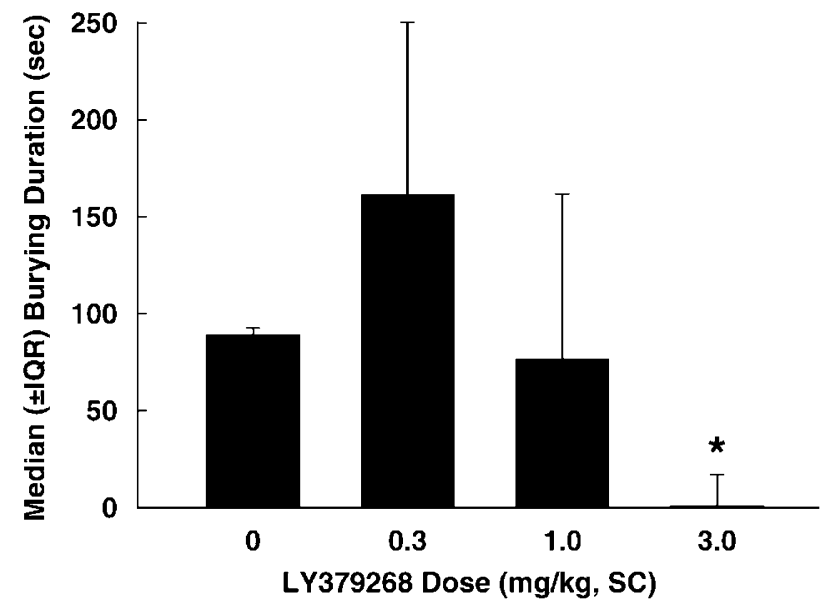

Figure 3 Time, in seconds, spent burying a previously electrified probe in the defensive burying test for animals pretreated with $0,0.3,1.0$, or $3.0 \mathrm{mg} /$ $\mathrm{kg}$ of LY379268 (*p $<0.05$ with a Mann-Whitney U-test vs vehicle).
Mann-Whitney $U$ pairwise comparisons revealed that at the $0.3 \mathrm{mg} / \mathrm{kg}$ dose LgA rats exhibited increased burying compared to the ShA $(U=2, p<0.05)$ and Ctrl $(U=4$, $p<0.05$ ) groups, while the ShA and Ctrl groups did not differ from one another. When examining the dose-response function collapsed across drug-history groups, Mann-Whitney $U$ pairwise comparisons of burying behavior $v s$ the LY329268naive animals from Experiment 1 revealed a significant reduction in burying only at the $3.0 \mathrm{mg} / \mathrm{kg}(U=2, p<0.05)$ dose, similar to that observed in Experiment 2.

Other behavioral measures. No significant effects were observed for freezing, shock reactivity, ambulation, or rearing in the LgA group vs the ShA and Ctrl groups at any time point. In addition, no differences in these behaviors were observed between ShA and Ctrl groups at any time point (data not shown).

\section{DISCUSSION}

The results extended understanding of the consequences of escalated cocaine intake to alterations in stress reactivity with findings that a history of cocaine escalation is associated with (1) increased shock probe-induced defen-

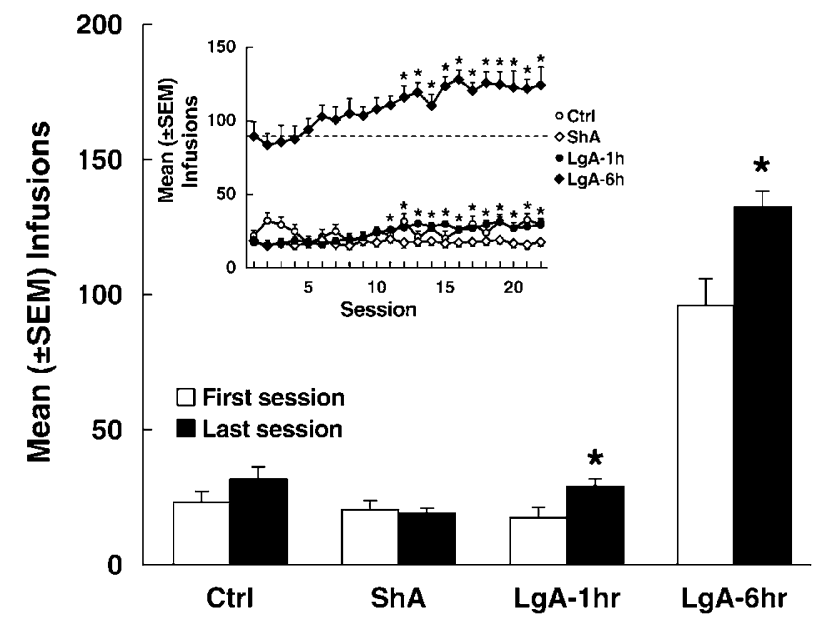

Figure 4 Number of lever presses on the first vs last self-administration session for food control, short access cocaine (ShA), and long access cocaine (LgA-I h, LgA-6h) rats in (* $p<0.05$ with a paired $t$-test on first vs last self-administration session). Inset: number of lever presses on each day of self-administration corresponding to the escalation phase of the experiment for Ctrl, ShA, LgA (I and 6 h) rats (*p $<0.05$ vs session I).

Table I Effect of LY379268 (mg/kg) on Duration (s) of Ambulation, Rearing and Freezing (Mean \pm SEM), and on Shock Reactivity (Median (Interquartile Range))

\section{Dose of LY379268 (mg/kg)}

\begin{tabular}{|c|c|c|c|c|c|c|}
\hline \multirow[b]{2}{*}{ Behavior } & & & & & \multirow[b]{2}{*}{ Test } & \multirow[b]{2}{*}{$p$} \\
\hline & 0 & 0.3 & $\mathbf{I}$ & 3 & & \\
\hline Ambulation & $125.2 \pm 9.7$ & $142.6 \pm 8.5$ & $153.5 \pm 5.3$ & $124.2 \pm 23.8$ & $F_{3,38}=0.95$ & 0.45 \\
\hline Rearing & $170.3 \pm 23.9$ & $158.2 \pm 15.3$ & $132.3 \pm 17.9$ & $93.39 \pm 29.4$ & $F_{3,38}=2.36$ & 0.09 \\
\hline Freezing & $26.5 \pm 12.0$ & $15.9 \pm 2.9$ & $42.1 \pm 10.6$ & $16.1 \pm 6.7$ & $F_{3,38}=1.73$ & 0.19 \\
\hline Shock reactivity & $2(0)$ & $2(0)$ & $2(0)$ & $2(0)$ & $\mathrm{H}_{3,38}=0.84$ & 0.83 \\
\hline
\end{tabular}




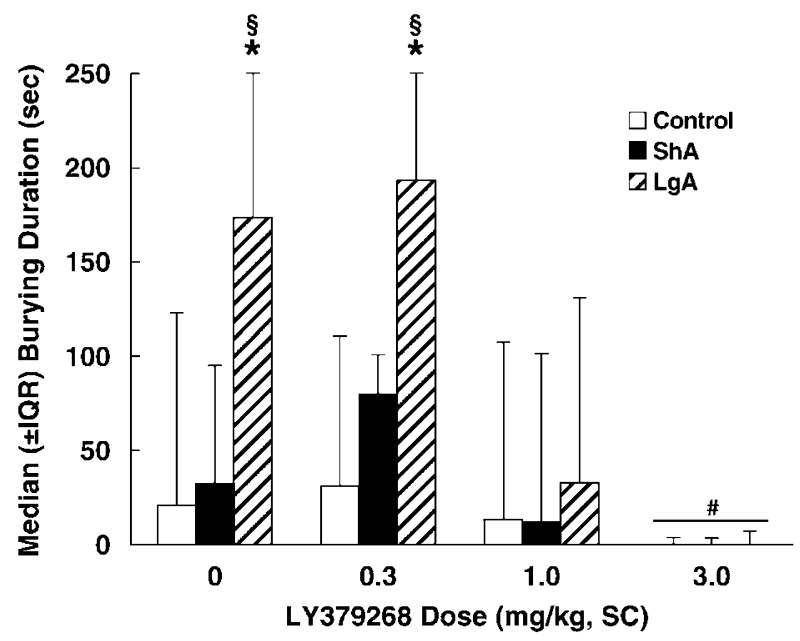

Figure 5 Mean time $( \pm$ SEM) spent burying a previously electrified probe in the defensive burying test for animals pretreated with $0.3,1.0$, or $3.0 \mathrm{mg} / \mathrm{kg}$ of LY379268 14 days from the last self-administration session (*p $<0.05$ with a Mann-Whitney U-test vs food controls (Ctrl), ${ }^{{ }^{\$}} p<0.05$ with a Mann-Whitney U-test vs short access cocaine (ShA), ${ }^{\#} p<0.05$ with a Mann-Whitney U-test vs LY372968-naive animals from Experiment I).

sive burying lasting up to 6 weeks following termination of drug intake and (2) sensitivity to the anxiolytic-like effects of the mGlu2/3 agonist LY379268 at a dose $(1.0 \mathrm{mg} / \mathrm{kg})$ that does not produce anxiolytic-like effects in animals with short daily cocaine access or drug-naive controls. Importantly, rats in the cocaine escalation groups did not show significantly different levels of ambulation or rearing at any abstinence time point, indicating that the increased burying behavior in these animals was not likely the result of a nonspecific increase in motor output. Furthermore, the observation that each experimental group exhibited similar startle responses upon first contact with the shock probe at all time points indicates that the increased burying in the cocaine-escalated rats was not an artifact of increased pain sensitivity.

The results confirm that chronic cocaine exposure, under conditions that lead to escalating cocaine intake, produces robust and long-lasting exacerbation of stress reactivity as reflected by increased defensive burying. This effect was not seen in rats exposed to cocaine under conditions that maintain stable cocaine self-administration as illustrated by the lack of differences in defensive burying between the ShA and drug-naive control groups with a similar history of reinforcement and daily handling.

In addition to the increased duration of defensive burying observed in the LgA group, the present data show that LY379268 strongly attenuated defensive burying in the $\operatorname{LgA}$ group and, to a lesser extent, also decreased defensive burying in the ShA and drug-naive groups. The finding that the LgA group, but not the ShA cocaine or cocaine-naive control groups, showed a reduction in burying at the $1.0 \mathrm{mg} / \mathrm{kg}$ dose of LY379268 suggests that these animals may be more sensitive to the anxiolytic-like effects of the mGlu2/ 3 agonist. This interpretation is supported by the observation that a reduction in anxiety-like behavior by LY379268 occurred in drug-naive animals only at the $3.0 \mathrm{mg} / \mathrm{kg}$ dose. Notably, the $3.0 \mathrm{mg} / \mathrm{kg}$ dose of LY379268 produced a nearly complete suppression of defensive burying across all access conditions. Similarly, rats without any reinforcement history (Experiment 2) exhibited decreased burying only at the $3.0 \mathrm{mg} / \mathrm{kg}$ dose. Thus, drug-naive food controls and the ShA cocaine group (Experiment 3) did not differ from drug and self-administration-naive controls (Experiment 2) with respect to the anxiolytic-like effects of LY379268. Moreover, the failure to observe any differences among the cocaine-naive food control group, the ShA cocaine and $\operatorname{LgA}$ cocaine groups with respect to locomotor output or pain sensitivity strongly argues against the possibility that the increased defensive burying in the $\operatorname{LgA}$ group was the result of nonspecific sedative or motor effects. Collectively, these observations indicate that rats with a history of escalated cocaine intake (ie a history leading to increased stress sensitivity in the defensive burying test) may have been behaviorally more sensitive to the anxiolytic-like effects of LY379268 than rats with a history of limited access to cocaine or drug-naive controls. Unequivocal confirmation of this interpretation will remain, however, for future studies because differences in the baseline duration of defensive burying existed among the different treatment groups. Thus, a possible alternative interpretation for the differential effects of LY379268 in the LgA vs ShA, and drugnaive control groups is that LY379268 may have acted with greater efficiency on the higher levels of defensive burying in the LgA group, compared to the low levels of burying in the other groups.

Rats in the ShA group, unlike those in the LgA group, did not differ from drug-naive controls in the degree of defensive burying at any time point. In the case of stressrelated behavioral output, it has been shown that rats with 48-h continuous access to cocaine exhibit increased stressinduced ultrasonic vocalizations, but this effect is significantly more pronounced in yoked controls receiving noncontingent cocaine infusions (Mutschler and Miczek, 1998b). Thus, under otherwise identical conditions, involuntary cocaine administration may produce greater stress reactivity during a drug-free period than voluntary selfadministration. This phenomenon may account for the failure of the ShA group to exhibit elevated levels of defensive burying $v s$ drug-naive controls in the present study, although increased defensive burying has been observed in rats following 3 days (Harris et al, 2001) or 2 weeks (Basso et al, 1999) of daily noncontingent (intraperitoneal) cocaine administration. Thus, it is possible that the failure to observe increased defensive burying in the ShA group may be due, in part, to the voluntary nature of cocaine administration.

Factors independent of cocaine-induced alterations in stress sensitivity may have contributed to the absence of between-group differences in defensive burying at the 84-day withdrawal time point. While there was no statistically significant difference among groups, it is important to note that rats in the $\operatorname{LgA}$ group at this time point did not show a significant reduction in burying compared to any earlier time point. Thus, while it cannot be concluded that the LgA group showed elevated stress reactivity $v s$ the ShA or drug-naive groups, one also cannot conclude that the increased stress reactivity associated with a history of long daily cocaine access had dissipated at this time point. To properly interpret the data at the 84-day withdrawal time point, it is important to note that increased 
variability in burying (compared to earlier withdrawal intervals) was observed for all of the groups, possibly reflecting age- and size-related effects on defensive burying behavior. Specifically, at this time point the increased size of the animals, paired with the limited space available in the testing apparatus, may have shifted the probability of engaging in passive behavioral stress responses such as freezing to an active behavioral response (ie burying) since passive avoidance of the probe becomes less feasible as space decreases. Support for this interpretation comes from previous reports indicating that the size of the defensive burying chamber is inversely related to the duration of burying in rats (Pinel et al, 1980). However, in the absence of specific controls, the contribution of age- and size-related effects to the present data remains speculative.

Evidence for changes in $\mathrm{mGlu} 2 / 3$ receptor function in animals with cocaine experience, albeit under nonescalation conditions, has been previously reported. For example, in the incubation model (Grimm et al, 2001), LY379268 given systemically or into the central nucleus of the amygdala (CeA) decreased cue-induced cocaine seeking 21 days but not 3 days following the termination of cocaine access ( $\mathrm{Lu}$ et al, 2007). While the CeA has been shown not to be involved in the control of defensive burying (Treit et al, 1993), it does play an important role in mediating other stress-related behaviors including stress-induced reinstatement (Erb et al, 2001; Leri et al, 2002) and conditioned fear (Killcross et al, 1997). Neuroadaptive changes in the pharmacological characteristics of $\mathrm{mGlu} 2 / 3$ receptors in the CeA may explain this differential effect of LY379268 following different periods of incubation. In a molecular study, desensitization of $m G l u 2 / 3$ receptors in the nucleus accumbens and PFChas been reported in rats 3 weeks following a 1-week regimen of noncontingent cocaine administration (Xi et al, 2002). The PFC has been implicated in the control of defensive burying (Shah and Treit, 2003, 2004), and changes in PFC mGlu2/3 function may be important with respect to the present findings. The precise implications of this finding, however, remain unclear. Escalation in the current study produced behavior consistent with increased sensitivity of $\mathrm{mGlu} 2 / 3$ receptors, whereas a desensitization of $m G l u 2 / 3$ receptors was found with limited noncontingent cocaine exposure. Nonetheless, overall, these findings suggest that neuroadaptive changes occur in mGlu2/3 function up to 3 weeks following the termination of cocaine, in regions that play a role in stressrelated behaviors, including defensive burying.

The septum is a structure that is known to play an important role in the regulation of defensive burying (Menard and Treit, 2001; Degroot and Treit, 2003, 2004) and that may show changes in glutamatergic function that contribute to the differential sensitivity to the effects of LY379268 on defensive burying observed in the LgA group. While this hypothesis has to date not been directly tested, some evidence may be gleaned from in vitro data. Specifically, enhanced glutamate release within the septum in slices from animals that had received 14 or 28 , but not 7 , days of chronic cocaine has been demonstrated (Shoji et al, 1997). Examination of the spontaneous inhibitory postsynaptic potentials revealed that this finding was the result of the diminished effectiveness of $\mathrm{GABA}_{\mathrm{B}}$ auto- and heteroreceptors that exert inhibitory control of postsynaptic glutamate release (Shoji et al, 1997). Moreover, evidence for a role of glutamatergic transmission, in particular, in mediating anxiety-related behaviors has been demonstrated with the finding that intra-septal infusions of the $\mathrm{N}$-methylD-aspartate receptor antagonist CNQX attenuate defensive burying and increase open-arm exploration on the elevated plus maze (Menard and Treit, 2001). These data reveal an important role for glutamatergic transmission in the septum specifically in defensive burying. Furthermore, a possible role for mGlu2/3 receptors is indicated by the observation that $\mathrm{c}$-fos induction is increased in the septum following pharmacological manipulation of glutamate transmission with the mGlu2/3 receptor agonist LY354740 and the mGlu2/3 receptor antagonist LY341495 (Linden et al, 2005b). Considering the present findings showing a differential effect of the anxiolytic-like effects of LY379268 in animals with differing drug histories, it will be important to determine in the future whether neuroadaptive changes in glutamatergic function occur in the septum, particularly at the level of mGlu2/3 receptors, in animals with a history of escalated cocaine intake.

The alterations in stress-reactivity in the $\operatorname{LgA}$ group are likely to have important implications for the initiation, maintenance, and relapse to cocaine use. Rats with a history of long $(10.5 \mathrm{~h})$ daily cocaine access are more susceptible to response-reinstating actions of a single noncontingent cocaine administration than rats maintained on limited (3.5 h) daily access to cocaine (Mantsch et al, 2004). Several findings are consistent with the hypothesis that the development of escalating cocaine intake may be due, in part, to stress-related mechanisms. Daily exposure to electric foot shock in cocaine self-administering rats under $2 \mathrm{~h}$ limited access conditions resulted in an escalated pattern of cocaine intake over 14 sessions (Mantsch and Katz, 2006), and exposure to social-defeat stress increased total cocaine self-administration during two 12-h binge-like sessions in rats (Covington and Miczek, 2001). These findings suggest that heightened sensitivity to stress as observed in the LgA group is likely to be an important factor contributing to progressive increases in the dose or amount of cocaine intake associated with cocaine addiction. The increased stress reactivity in animals with a history of cocaine escalation also has implications for vulnerability to stress-induced relapse. Stressful imagery has been reported to elicit drug craving in abstinent cocaine users (Fox et al, 2005). Moreover, using the same procedure, an inverse relationship between stress-induced cocaine craving and time to relapse, as well as a positive relationship between stress-induced cortisol levels and drug intake, during relapse, has been reported (Sinha et al, 2006). Data from animal models of relapse also support the importance of stress in reinstating previously extinguished drug-seeking behavior (see Shaham et al, 2000 for a review) and, in the case of heroin, rats with long (11-h) access exhibit greater levels of stress-induced reinstatement compared to those with short (1-h) access (Ahmed et al, 2000). Thus, increased and persistent stress reactivity associated with a history of escalated cocaine self-administration suggests that increased sensitivity to stress induced by chronic cocaine may exacerbate the role of stress per se as a risk factor for relapse. Moreover, because stress also induces craving (Fox et $a l, 2005)$, a condition that has been shown to be 
predictive of shorter time to relapse and increased drug intake during relapse (Sinha et al, 2006), increased stress reactivity may further exacerbate the likelihood and severity of relapse associated with cocaine craving.

In summary, the present observations support the hypothesis that chronic cocaine use produces a pronounced and enduring exacerbation of stress reactivity that may play a significant role in cocaine addiction, including the maintenance of ongoing self-administration, withdrawal severity, and susceptibility to stress-induced relapse. These findings also illustrate the importance of developing therapeutic interventions aimed at reducing stress as a strategy for reducing relapse risk. Consistent with recent reports showing a reduction in the response-reinstating effects of cocaine (Peters and Kalivas, 2006; Adewale et al, 2006), drug-associated stimuli (Baptista et al, 2004; Bossert et al, 2004, 2006; Zhao et al, 2006), and stressful stimuli (Zhao et al, 2006), the present data indicate that mGlu2/3 receptors may be promising treatment targets.

\section{ACKNOWLEDGEMENTS}

This is publication 18380-MIND from The Scripps Research Institute and is supported by NIDA grant DA 017097 and DA 08467 (FW). We thank Dr CV Dayas, Dr Y Zhao, and Dr N Sidhpura for their comments and Michael Arends for editorial assistance.

\section{DISCLOSURE}

None of the authors have any relevant financial interests to disclose or any conflict of interests.

\section{REFERENCES}

Adewale AS, Platt DM, Spealman RD (2006). Pharmacological stimulation of group II metabotropic glutamate receptors reduces cocaine self-administration and cocaine-induced reinstatement of drug seeking in squirrel monkeys. J Pharmacol Exp Ther 318: 922-931.

Ahmed SH, Kenny PJ, Koob GF, Markou A (2002). Neurobiological evidence for hedonic allostasis associated with escalating cocaine use. Nat Neurosci 5: 625-626.

Ahmed SH, Koob GF (1998). Transition from moderate to excessive drug intake: change in hedonic set point. Science 282: 298-300.

Ahmed SH, Koob GF (1999). Long-lasting increase in the set point for cocaine self-administration after escalation in rats. Psychopharmacology 146: 303-312.

Ahmed SH, Walker JR, Koob GF (2000). Persistent increase in the motivation to take heroin in rats with a history of drug escalation. Neuropsychopharmacology 22: 413-421.

Antelman SM, Eichler AJ, Black CA, Kocan D (1980). Interchangeability of stress and amphetamine in sensitization. Science 207: 329-331.

Baptista MAS, Martin-Fardon R, Weiss F (2004). Preferential effects of the metabotropic glutamate $2 / 3$ receptor agonist LY379268 on conditioned reinstatement $v s$ primary reinforcement: comparison between cocaine and a potent conventional reinforcer. J Neurosci 24: 4723-4727.

Basso AM, Spina M, Rivier J, Vale W, Koob GF (1999). Corticotropin-releasing factor antagonist attenuates the 'anxiogenic-like' effect in the defensive burying paradigm but not in the elevated plus-maze following chronic cocaine in rats. Psychopharmacology 145: 21-30.

Bossert JM, Gray SM, Lu L, Shaham Y (2006). Activation of group II metabotropic glutamate receptors in the nucleus accumbens shell attenuates context-induced relapse to heroin seeking. Neuropsychopharmacology 31: 2197-2209.

Bossert JM, Liu SY, Lu L, Shaham Y (2004). A role of ventral tegmental area glutamate in contextual cue-induced relapse to heroin seeking. J Neurosci 24: 10726-10730.

Bozarth MA, Wise RA (1985). Toxicity associated with long-term intravenous heroin and cocaine self- administration in the rat. J Am Med Assoc 254: 81-83.

Breese GR, Chu K, Dayas CV, Funk D, Knapp DJ, Koob GF et al (2005). Stress enhancement of craving during sobriety: a risk for relapse. Alcohol Clin Exp Res 29: 185-195.

Caine SB, Koob GF (1993). Modulation of cocaine self-administration in the rat through D-3 dopamine receptors. Science 260: 1814-1816.

Covington III HE, Miczek KA (2001). Repeated social-defeat stress, cocaine or morphine. Effects on behavioral sensitization and intravenous cocaine self-administration 'binges'. Psychopharmacology 158: 388-398.

De Boer SF, Koolhaas JM (2003). Defensive burying in rodents: ethology, neurobiology and psychopharmacology. Eur J Pharmacol 463: 145-161.

Degroot A, Treit D (2003). Septal GABAergic and hippocampal cholinergic systems interact in the modulation of anxiety. Neuroscience 117: 493-501.

Degroot A, Treit D (2004). Anxiety is functionally segregated within the septo-hippocampal system. Brain Res 1001: 60-71.

Erb S, Salmaso N, Rodaros D, Stewart J (2001). A role for the CRFcontaining pathway from central nucleus of the amygdala to bed nucleus of the stria terminalis in the stress-induced reinstatement of cocaine seeking in rats. Psychopharmacology 158: 360-365.

Erb S, Shaham Y, Stewart J (1996). Stress reinstates cocaineseeking behavior after prolonged extinction and a drug-free period. Psychopharmacology 128: 408-412.

Fox HC, Talih M, Malison R, Anderson GM, Kreek MJ, Sinha R (2005). Frequency of recent cocaine and alcohol use affects drug craving and associated responses to stress and drug-related cues. Psychoneuroendocrinology 30: 880-891.

Grimm JW, Hope BT, Wise RA, Shaham Y (2001). Neuroadaptation. Incubation of cocaine craving after withdrawal. Nature 412: 141-142.

Harris GC, Altomare K, Aston-Jones G (2001). Preference for a cocaine-associated environment is attenuated by augmented accumbal serotonin in cocaine withdrawn rats. Psychopharmacology (Berl) 156: 14-22.

Johanson CE, Balster RL, Bonese K (1976). Self-administration of psychomotor stimulant drugs: the effects of unlimited access. Pharmacol Biochem Behav 4: 45-51.

Killcross S, Robbins TW, Everitt BJ (1997). Different types of fearconditioned behaviour mediated by separate nuclei within amygdala. Nature 388: 377-380.

Leri F, Flores J, Rodaros D, Stewart J (2002). Blockade of stressinduced but not cocaine-induced reinstatement by infusion of noradrenergic antagonists into the bed nucleus of the stria terminalis or the central nucleus of the amygdala. J Neurosci 22: 5713-5718.

Linden AM, Bergeron M, Schoepp DD (2005b). Comparison of cFos induction in the brain by the mGlu2/3 receptor antagonist LY341495 and agonist LY354740: Evidence for widespread endogenous tone at brain mGlu2/3 receptors in vivo. Neuropharmacology 49(Suppl 1): 120-134.

Linden AM, Shannon H, Baez M, Yu JL, Koester A, Schoepp DD (2005a). Anxiolytic-like activity of the mGLU2/3 receptor agonist LY354740 in the elevated plus maze test is disrupted in metabotropic glutamate receptor 2 and 3 knock-out mice. Psychopharmacology 179: 284-291. 
Lorrain DS, Baccei CS, Correa LD, Bristow LJ (2005). Comparison of the effects of diazepam, the CRF1 antagonist CP-154,526 and the group II mGlu receptor agonist LY379268 on stress-evoked extracellular norepinephrine levels. Neuropharmacology 48: 927-935.

Lu L, Uejima JL, Gray SM, Bossert JM, Shaham Y (2007). Systemic and central amygdala injections of the mGluR2/3 agonist LY379268 attenuate the expression of incubation of cocaine craving. Biol Psychiatry 61: 591-598.

Mantsch JR, Katz ES (2006). Elevation of glucocorticoids is necessary but not sufficient for the escalation of cocaine selfadministration by chronic electric footshock stress in rats. Neuropsychopharmacology 32: 367-376.

Mantsch JR, Yuferov V, Mathieu-Kia AM, Ho A, Kreek MJ (2004). Effects of extended access to high $v s$ low cocaine doses on selfadministration, cocaine-induced reinstatement and brain mRNA levels in rats. Psychopharmacology 175: 26-36.

Markou A, Koob GF (1991). Postcocaine anhedonia. An animal model of cocaine withdrawal. Neuropsychopharmacology 4: 17-26.

Meaney MJ, Brake W, Gratton A (2002). Environmental regulation of the development of mesolimbic dopamine systems: a neurobiological mechanism for vulnerability to drug abuse? Psychoneuroendocrinology 27: 127-138.

Menard J, Treit D (2001). The anxiolytic effects of intrahippocampal midazolam are antagonized by intra-septal L-glutamate. Brain Res 888: 163-166.

Mutschler NH, Miczek KA (1998a). Withdrawal from i.v. cocaine 'binges' in rats: ultrasonic distress calls and startle. Psychopharmacology 135: 161-168.

Mutschler NH, Miczek KA (1998b). Withdrawal from a selfadministered or non-contingent cocaine binge: differences in ultrasonic distress vocalizations in rats. Psychopharmacology 136: 402-408.

Pesold C, Treit D (1992). Excitotoxic lesions of the septum produce anxiolytic effects in the elevated plus-maze and the shock-probe burying tests. Physiol Behav 52: 37-47.

Peters J, Kalivas PW (2006). The group II metabotropic glutamate receptor agonist, LY379268, inhibits both cocaine- and foodseeking behavior in rats. Psychopharmacology 186: 143-149.

Pinel JPJ, Treit D, Ladak F, Maclennan J (1980). Conditioned defensive burying in rats free to escape. Anim Learn Behav 8: 447-451.

Pinel JPJ, Treit D (1978). Burying as a defensive response in rats. J Comp Physiol Psychol 92: 708-712.

Post RM, Rose H (1976). Increasing effects of repetitive cocaine administration in the rat. Nature 260: 731-732.

Prasad BM, Sorg BA, Ulibarri C, Kalivas PW (1995). Sensitization to stress and psychostimulants. Involvement of dopamine transmission $v$ s the HPA axis. Ann NY Acad Sci 771: 617-625.
Shah AA, Treit D (2003). Excitotoxic lesions of the medial prefrontal cortex attenuate fear responses in the elevated plusmaze, social interaction, and shock probe burying tests. Brain Res 969: 183-194.

Shah AA, Treit D (2004). Infusions of midazolam into the medial prefrontal cortex produce anxiolytic effects in the elevated plusmaze and shock-probe burying tests. Brain Res 996: 31-40.

Shaham Y, Erb S, Stewart J (2000). Stress-induced relapse to heroin and cocaine seeking in rats: a review. Brain Res Brain Res Rev 33: 13-33.

Shoji S, Simms D, McDaniel WC, Gallagher JP (1997). Chronic cocaine enhances $\gamma$-aminobutyric acid and glutamate release by altering presynaptic and not postsynaptic $\gamma$-aminobutyric acid receptors within the rat dorsolateral septal nucleus. J Pharmacol Exp Ther 280: 129-137.

Sinha R (2001). How does stress increase risk of drug abuse and relapse? Psychopharmacology 158: 343-359.

Sinha R, Garcia M, Paliwal P, Kreek MJ, Rounsaville BJ (2006). Stress-induced cocaine craving and hypothalamic-pituitaryadrenal responses are predictive of cocaine relapse outcomes. Arch Gen Psychiatry 63: 324-331.

Sorge RE, Stewart J (2005). The contribution of drug history and time since termination of drug taking to footshock stress-induced cocaine seeking in rats. Psychopharmacology 183: 210-217.

Treit D, Pesold C, Rotzinger S (1993). Dissociating the anti-fear effects of septal and amygdaloid lesions using two pharmacologically validated models of rat anxiety. Behav Neurosci 107: 770-785.

Weiss F, Maldonado-Vlaar CS, Parsons LH, Kerr TM, Smith DL, Ben-Shahar O (2000). Control of cocaine-seeking behavior by drug-associated stimuli in rats: effects on recovery of extinguished operant-responding and extracellular dopamine levels in amygdala and nucleus accumbens. Proc Natl Acad Sci USA 97: $4321-4326$.

Xi Z-X, Ramamoorthy S, Baker DA, Shen H, Samuvel DJ, Kalivas PW (2002). Modulation of group II metabotropic glutamate receptor signaling by chronic cocaine. J Pharmacol Exp Ther 303: 608-615.

Yokel RA, Pickens R (1973). Self-administration of optical isomers of amphetamine and methylamphetamine by rats. J Pharmacol Exp Ther 187: 27-33.

Zhao Y, Dayas CV, Aujla H, Baptista MAS, Martin-Fardon R, Weiss F (2006). Activation of group II metabotropic glutamate receptors attenuates both stress and cue-induced ethanol seeking and modulates c-fos expression in the hippocampus and amygdala. J Neurosci 26: 9967-9974. 